\title{
Caratterizzazione della proteinuria nelle glomerulonefriti mediante elettroforesi in gel di poliacrilamide (SDS-PAGE): significato clinico dei pattern proteinurici
}

\author{
C. Bazzi ${ }^{1}$, C. Petrini' ${ }^{2}$, V. Rizza ${ }^{2}$ \\ ${ }^{1}$ Divisione di Nefrologia e ${ }^{2}$ Laboratorio di Biochimica \\ Azienda Ospedaliera "Ospedale San Carlo Borromeo" - Milano
}

elle principali forme di glomerulonefrite $(\mathrm{GN})$ primitiva [glomerulosclerosi focale (GSF), glomerulonefrite membranosa (GNM), glomerulonefrite membrano-proliferativa (GNMP), glomerulonefrite a depositi mesangiali di $\operatorname{IgA}(\operatorname{Ig} \mathrm{AN})]$ la progressione alla insufficienza renale cronica (IRC) è significativamente associata alla entità della proteinuria (1-4). Nelle stesse forme di GN la progressione è anche significativamente associata all'estensione delle alterazioni tubulo-interstiziali, più che alla entità delle alterazioni glomerulari (5-8). Una eccezione alla regola della associazione tra proteinuria e progressione è costituita dalla $\mathrm{GN}$ a lesioni minime (GNLM) nella quale livelli di proteinuria paragonabili a quelli che si osservano, per esempio, nella GSF non si associano alla tendenza alla progressio- ne. Varie ipotesi sono state formulate per rendere conto di questa differenza di comportamento tra GNLM e le altre forme di GN caratterizzate dalla presenza di sindrome nefrosica: nella GNLM la proteinuria è selettiva e, in genere, di breve durata per la elevata steroido-sensibilità; nella GNLM inoltre non si riscontrano le alterazioni tubulo-interstiziali, frequentemente presenti nelle altre forme di GN primitiva (9).

Attualmente si ritiene che esista una relazione patogenetica tra proteinuria e alterazioni-tubulo interstiziali, attraverso una possibile azione "tubulo-tossica" della proteinuria. In effetti è stato documentato che le cellule tubulari, in risposta a vari stimoli, uno dei quali può essere rappresentato dalla proteinuria, producono citochine, fattori di crescita e sostanze vasoattive in grado a loro volta di promuovere infiltrazione interstiziale di linfo-monociti, proliferazione dei fibroblasti residenti con produzione di matrice extracellulare e conseguente fibrosi interstiziale e alterazioni vascolari (rassegna in [9]). L'ipotesi di una relazione tra proteinuria e danno tubulo-interstiziale è stata avvalorata da numerosi modelli sperimentali di glomerulonefrite, nei quali è stata documentata non solo una associazione tra proteinuria e alterazioni tubulo-interstiziali, ma anche una attenuazione delle alterazioni tubulo-interstiziali in seguito a manovre terapeutiche idonee a ridurre la proteinuria (dieta ipoproteica, ACE-inibitori, etc.) (4). Una associazione tra proteinuria e danno tubulo-interstiziale è stata evidenziata in alcune GN spontanee umane come la GNM (10).

Nonostante questo ruolo centrale ai fini 
della progressione assegnato alla proteinuria ed alle alterazioni tubulo-interstiziali e nonostante la attuale disponibilità di metodiche di laboratorio idonee a evidenziare la componente tubulare della proteinuria, assai scarsi sono stati finora gli studi finalizzati a caratterizzare nelle GN la componente tubulare della proteinuria per definirne significato clinico, associazione con alterazioni istologiche, valore predittivo rispetto alla progressione e alla risposta alla terapia.

Scopo di questa rassegna è quello di illustrare sinteticamente le caratteristiche di uno dei principali metodi di caratterizzazione della proteinuria, la elettroforesi in gel di poliacrilamide (SDS-PAGE) e di esaminare la letteratura disponibile sul significato clinico dei pattern SDS-PAGE nelle GN.

\section{Fisiopatologia della filtrazione glomerulare e del riassorbimento tubulare delle proteine}

La parete del capillare glomerulare in condizioni fisiologiche costituisce una barriera al passaggio delle proteine di peso molecolare uguale o superiore a quello dell'albumina, mentre le proteine di peso molecolare inferiore a quello della albumina sono liberamente filtrate e quasi completamente riassorbite dall'epitelio del tubulo prossimale. L'accertamento delle caratteristiche di selettività alle proteine della parete del capillare glomerulare è stato raggiunto mediante lo studio, sia nell'uomo che nell' animale da esperimento, delle clearances frazionali di varie macromolecole esogene, neutre o caricate elettricamente, dallo studio della localizzazione a livello della parete capillare di vari traccianti a diversa massa molecolare e dallo studio delle clearances frazionali di macromolecole esogene a differente configurazione spaziale. Sulla base dei risultati di queste indagini si è arrivati alla conclusione che la parete del capillare glomerulare ostacola il passaggio delle proteine plasmatiche in base a meccanismi che dipendono dalle dimensioni, dalla carica elettrica e dalla configurazione spaziale delle molecole proteiche ed è stata definita una teoria della configurazione della parete capillare glomerulare $(11,12)$. In base a ta- le teoria la parete del capillare glomerulare si comporta come una membrana caratterizzata dalla presenza di pori cilindrici di diametro differente. Sono stati ipotizzati tre possibili modelli di configurazione della parete del capillare glomerulare: il modello che gode di maggior credito, perché si adatta con maggiore precisione alle curve di escrezione delle macromolecole di destrano osservate in condizioni patologiche, è il cosiddetto "log-normal + shunt model": esso presuppone che la maggior parte della parete del capillare glomerulare sia perforata da una popolazione di pori cilindrici che hanno una distribuzione continua di tipo log-normale del loro raggio (raggio medio $53.2 \AA$ ); esiste, inoltre, una seconda popolazione di pori a minore densità e a raggio maggiore, che lascia passare le molecole di destrano fino a un raggio di $60 \AA$ e che funziona come una "shunt pathway" parallela (raggio medio dei pori della "shunt pathway" > $80 \AA$ ); l'area occupata da questi pori di maggiori dimensioni, assai limitata in condizioni normali, aumenta molto in condizioni patologiche e rende conto delle caratteristiche di non selettività della proteinuria in molte nefropatie glomerulari.

La barriera al passaggio delle molecole proteiche dipende inoltre dalla loro carica elettrica, nel senso che, in conseguenza della carica elettrica negativa sia della membrana basale glomerulare che del glicocalice che riveste le cellule endoteliali ed epiteliali, viene ostacolato il passaggio delle proteine elettronegative. Infine anche la configurazione spaziale delle proteine condiziona il loro passaggio attraverso i pori della membrana basale glomerulare, in quanto a parità di raggio, è facilitato il passaggio delle proteine con configurazione spiraliforme e con struttura flessibile rispetto a quelle a configurazione sferica e a struttura rigida.

Le proteine plasmatiche presenti nel filtrato glomerulare vengono riassorbite dalle cellule del tubulo prossimale (13, 14). Il riassorbimento avviene con un meccanismo di endocitosi da parte delle cellule tubulari prossimali, preceduto da un legame delle proteine alla membrana luminale; tale legame è regolato da recettori presenti sulla membrana luminale; esiste una grande variabilità nella affinità delle diverse proteine per la membrana luminale, dipendente dal numero e dalla struttura chimica dei "binding sites" della membrana e dalla carica elet- trica netta delle molecole proteiche. Il processo di endocitosi sembra essere largamente specifico quando la quantità di proteine da riassorbire è piccola, mentre è molto meno specifico quando tale quantità è elevata. Le proteine riassorbite sono inizialmente concentrate in vescicole (fagosomi, endosomi) che successivamente si fondono con i lisosomi intracitoplasmatici, dove le proteine vengono idrolizzate dagli enzimi lisosomiali ritornando in circolo sotto forma di aminoacidi. Se la quantità di proteine da riassorbire supera la capacità tubulare massima di riassorbimento, parte delle proteine non vengono riassorbite e si ritrovano in quantità dosabili nelle urine. Inoltre quando si verifica una sproporzione tra entità del riassorbimento ed entità del catabolismo intra-lisosomiale, si verifica un accumulo di proteine all'interno della cellula tubulare con conseguente danno cellulare.

\section{Elettroforesi in gel di poliacrilamide}

Per lo studio della composizione qualitativa della proteinuria, utile per identificare la localizzazione nel nefrone del danno anatomico e/o funzionale responsabile della proteinuria, sono disponibili varie tecniche separative: le tecniche elettroforetiche, nelle varie e successive versioni, sono state quelle che hanno avuto maggiore diffusione e applicazione clinica. Come è noto, utilizzando supporti qualì l'acetato di cellulosa, il nitrato di cellulosa e il gel di agarosio, la separazione delle particelle proteiche avviene sulla base delle differenze di carica elettrica netta esistenti tra le varie proteine, con formazione di zone costituite da proteine aventi uguale carica elettrica.

L'introduzione di supporti con le caratteristiche fisico-chimiche di setaccio molecolare, come il gel di poliacrilamide, consente di separare i componenti molecolari di miscele complesse di proteine sulla base della loro massa molecolare. Per la separazione delle proteine urinarie la variante della elettroforesi in gel di poliacrilamide che si avvale della aggiunta di sodio-dodecil-solfato (sodium dodecyl sulphate-poliacrylamide gel electrophoresis: SDS-PAGE) si è rivelata la più adeguata ed è stata introdotta da Pesce nel 1972 (15). Il pre-trattamento 
del campione con sodio-dodecil-solfato (SDS) consente di mascherare le cariche elettriche intrinseche della molecola proteica, in quanto l'SDS si lega fortemente alla catena polipeptidica nel rapporto di circa una molecola di SDS ogni due aminoacidi della proteina. Ogni molecola di SDS possiede una carica elettrica negativa e conferisce pertanto alla proteina un elevato numero di cariche elettriche negative, di gran lunga in eccesso rispetto alle cariche intrinseche della proteina stessa. Pertanto, il rapporto carica/massa è virtualmente costante per tutte le proteine e il processo di separazione può attuarsi soltanto sulla base dell'effetto di setaccio esercitato dalla dimensione dei pori del gel e quindi in dipendenza del raggio molecolare. Pertanto le proteine che migrano lungo una matrice di poliacrilamide a maglie trasversali vengono più o meno rallentate in base alle loro dimensioni e si distribuiscono in bande costituite da proteine omogenee per dimensione. Il supporto di gel di poliacrilamide può essere preparato in forma di colonna (di variabile diametro) o di lastra, di variabile spessore; inoltre si possono allestire gel a concentrazione omogenea o in gradiente di concentrazione di acrilamide. I supporti con gradiente di concentrazione sono i più adatti alla separazione di miscele proteiche complesse come le urine. Per calcolare la massa molecolare delle proteine separate ci si avvale di miscele di proteine di massa molecolare nota, che vengono fatte migrare contemporaneamente ai campioni in esame.

Per la rivelazione delle proteine si utilizzano coloranti molto sensibili come il Coomassie Brilliant Blue R 250 o il nitrato d'argento o la doppia colorazione con Coomassie e Silver; tali colorazioni consentono di visualizzare tracciati anche utilizzando campioni urinari aventi una concentrazione proteica ancora nell' ambito della norma e quindi senza sottoporre i campioni in esame a un processo di concentrazione, con gli inerenti inconvenienti. Sui tracciati elettroforetici ottenuti con l'SDS-PAGE è possibile procedere ad una identificazione delle proteine contenute nelle varie bande, applicando la tecnica dell'immunoblotting, con trasferimento delle bande dal gel di poliacrilamide a un supporto di nitrocellulosa e identificazione delle varie proteine mediante l'impiego di anticorpi specifici. Da alcuni anni sono disponibili in commercio delle micro-piastre preformate
(Phast ® System, Pharmacia, Upsala, Sweden) che devono essere utilizzate su un apposito sistema elettroforetico, completo di modulo per la colorazione automatica dei gel. I risultati ottenuti con il Phast System secondo Lison et al (16) e Stierle et al (17) sono paragonabili a quelli che si ottengono con il macrometodo (da noi utilizzato) e consentono un notevole risparmio del tempo di lavoro. Sulla base dei tracciati proteici urinari ottenuti con l'SDS-PAGE sono stati definiti vari sistemi di classificazione. Una recente versione di questo tipo di classificazione (17) ha identificato 6 pattern di proteinuria renale (Tab. I): I) glomerulare non selettiva; II) glomerulare a media selettività; III) glomerulare selettiva; IV) tubulare; Va) mista glomerulo-tubulare; Vb) mista tubulo-glomerulare; VI) vascolare. Nei nostri studi noi abbiamo utilizzato la metodica di Laemmly (18) e abbiamo definito (19) una classificazione leggermente diversa che distingue 6 pattern di proteinuria renale (Fig. 1 e Tab. II): 1) fisiologica (solo albumina e talora proteina di Tamm Horsfall); 2) glomerulare non selettiva (proteine con peso molecolare (PM) compreso tra 70 e $>150 \mathrm{kD}$ ); 3) glomerulare selettiva [proteine con PM compreso tra 70 e 80 (albumina e transferrina)]; 4) tubulare (proteine con PM tra 70 e $10 \mathrm{kD}$ ); 5) mista glomerulotubulare incompleta (proteine con PM compreso tra $>150$ e $23 \mathrm{kD}$ ); 6) mista glomerulo-tubulare completa (con proteine di PM compreso tra $>150$ e $10 \mathrm{kD}$ ).

\section{Applicazione clinica dell'SDS-PAGE nelle glomerulonefriti}

I primi studi che hanno impiegato l'elettroforesi in gel di poliacrilamide per la caratterizzazione della proteinuria risalgono ai primi anni '70 (20) e sono stati principalmente finalizzati a verificare la utilità di questa metodica nella differenziazione tra nefropatie glomerulari e nefropatie tubulari.

È stato solo a partire dai primi anni '90 che la tecnica SDS-PAGE è stata impiegata nello studio di casistiche sufficientemente ampie di pazienti con glomerulonefriti primitive o secondarie, per valutare la presenza e le caratteristiche della componente tubulare della proteinuria ed accertarne il possibile significato clinico. Nagy et al (21) hanno studiato con SDSPAGE 45 pazienti con IgAN, valutando in particolare la presenza di proteine a basso peso molecolare (low molecular weight proteins [LMW]: $\mathrm{PM}<60 \mathrm{kD}$ ). La presenza di tali proteine, che si riscontrava in 29 su 45 pazienti, si correlava in maniera altamente significativa con i livelli di creatinina sierica e di proteinuria totale e con l'entità del danno tubulo-interstiziale valutato semiquantitativamente.

Woo et al (22), utilizzando il Phast System, hanno studiato 60 pazienti con IgAN, valutandone l'outcome funzionale

\section{TAB. I - CLASSIFICAZIONE DELLA PROTEINURIA RENALE IN BASE AL PATTERN SDS-PAGE}

$\begin{array}{lcc}\text { Tipo } & \begin{array}{c}\text { Range di massa molecolare } \\ (\mathbf{k D})\end{array} & \begin{array}{c}\text { Tipo di proteinuria in base a } \\ \text { pattern SDS-PAGE }\end{array} \\ \text { I } & 41-400 & \begin{array}{c}\text { Glomerulare non selettiva } \\ \text { II }\end{array} \\ \text { III } & 69-156 & \text { Glomerulare a media selettività } \\ \text { IV } & 41-90(+130) & \text { Glomerulare selettiva } \\ \text { Va } & 11-69(+\mathrm{TH}) & \text { Tubulare } \\ \mathrm{Vb} & 11-312 & \text { Glomerulo-Tubulare } \\ \mathrm{VI} & 11-312 & \text { Tubulo-Glomerulare }\end{array}$

(sec. Stierle, Oser e Boesken, 1990) 
TAB. II - CLASSIFICAZIONE DELLA PROTEINURIA RENALE IN BASE AL PATTERN SDS-PAGE

\begin{tabular}{lcc}
\hline Tipo & $\begin{array}{c}\text { Range di massa molecolare } \\
(\mathbf{k D})\end{array}$ & $\begin{array}{c}\text { Tipo di proteinuria in base a } \\
\text { pattern SDS-PAGE }\end{array}$ \\
\hline 1 & Tracce di albumina $(+\mathrm{TH})$ & Fisiologica \\
2 & $70->150$ & $\begin{array}{c}\text { Glomerulare non selettiva } \\
3\end{array}$ \\
4 & $70-80(+\mathrm{TH})$ & Glomerulare selettiva \\
5 & $10-70$ & Tubulare \\
6 & $23->150$ & Mista incompleta \\
\hline
\end{tabular}

(sec. Petrini, Rizza, Bazzi, 1995) dopo 6 anni. Questi Autori hanno definito 4 pattern proteinurici: due pattern glomerulari caratterizzati dalla presenza solo di proteine di medio peso molecolare (MMW) o di proteine di medio ed alto peso molecolare (MMW+HMW) e due pattern misti glomerulo-tubulari, caratterizzati dalla presenza di proteine anche di basso peso molecolare (LMW: $<69$ kD) pattern MMW+LMW e pattern HMW+MMW+LMW. I pazienti con pattern proteinurico caratterizzato dalla presenza di proteine LMW (presenti in 19 su 60 pazienti) avevano livelli di proteinuria totale e di creatinina sierica significativamente più elevati dei pazienti senza proteine LMW, ma soprattutto presentavano dopo 6 anni una percentuale di progressione alla insufficienza renale cronica significativamente più elevata (pazienti con proteine LMW: IRC 47\%; pazienti senza proteine LMW: IRC 10\%; $\mathrm{p}=0.001)$ (Tab. III).

Bazzi et al (23) hanno studiato con SDSPAGE 40 pazienti affetti da glomerulonefrite lupica (6 pazienti di classe III, 24 di classe IV, 10 di classe V WHO). Sono stati definiti 5 pattern proteinurici: un pattern fisiologico (solo albumina); 2 pattern glomerulari puri [selettivo (solo proteine di medio peso molecolare: MMW) e non selettivo (proteine MMW e HMW)] e 2 pattern misti: uno con proteine LMW fino a $40 \mathrm{kD}$ e uno con proteine LMW fino a $10 \mathrm{kD}$. I pattern misti erano più frequenti (33 su 40 pazienti) e presentavano una significativa correlazione con la infiltrazione interstiziale e con la sclerosi glomerulare e una correlazione non significativa con la creatinina sierica.

In un'ampia casistica di 145 pazienti con glomerulonefriti primitive (GSF 43, GNM 72, GNMP 30) Bazzi et al (24) hanno studiato le caratteristiche della proteinuria mediante SDS-PAGE, identificando 4 pattern proteinurici: un pattern fisiologico (solo albumina), un pattern glomerulare puro (proteine MMW e HMW) e 2 pattern misti, glomerulo-tubulari, uno con proteine HMW, MMW e LMW con peso molecolare fino a $23 \mathrm{kD}$ (pattern denominato $23 \mathrm{kD}$ ) e uno con proteine HMW, MMW e LMW con peso molecolare fino a $20-10 \mathrm{kD}$ (denominato pattern $10 \mathrm{kD}$ ). Il pattern fisiologico era presente nello $0.7 \%$ dei pazienti (pazienti studiati nella fase di remissione clinica completa), il pattern glomerulare puro era presente nel $1.4 \%$ dei pazienti; di
Fig. 1 - Pattern proteinurici SDS-PAGE
Linea 1: proteinuria fisiologica (solo albumina e monomero della proteina di Tamm-Horsfall);

Linea 2: proteinuria glomerulare non selettiva (proteine ad alto peso molecolare - $\mathrm{HMW}$ - e a medio peso molecolare - $M M W$ );

Linea 3: proteinuria mista glomerulo-tubulare con proteine $H M W, M M W$ e a basso peso molecolare - LMW fino a $45 \mathrm{kD}$;

Linea 4: proteinuria mista glomerulo-tubulare con proteine $H M W, M M W$ e LMW fino a $23 \mathrm{kD}$ (i pattern delle linee 3 e 4 sono stati raggruppati nel pattern denominato " $23 \mathrm{kD}$ ");

Linea 5: proteinuria mista glomerulo-tubulare con proteine $H M W, M H W$ e LMW fino a $12 \mathrm{kD}$ (pattern denominato " $10 \mathrm{kD}$ "):

Line St: proteine standard di peso molecolare noto $(94,67,43,30,20,12 \mathrm{kD})$. 
gran lunga più frequenti erano i pattern misti: il pattern $23 \mathrm{kD}$ era presente nel $61 \%$ e il $10 \mathrm{kD}$ nel $37 \%$ dei pazienti. I due pattern misti sono stati confrontati rispetto a vari parametri clinici ed istologici. Il pattern misto $10 \mathrm{kD}$ era significativamente associato a livelli più elevati di creatinina sierica $(\mathrm{p}<0.0001)$, di proteinuria totale $(\mathrm{p}=0.007)$ e a un grado più marcato di lesioni tubulo-interstiziali $(\mathrm{p}=0.015)$. L'outcome funzionale è stato valutato in 82 pazienti con funzionalità renale basale normale (creatininemia basale: $1.00 \pm 0.22 \mathrm{mg} / \mathrm{dl}$ ) e con un follow up di $46 \pm 22$ mesi confrontando 64 pazienti con pattern $23 \mathrm{kD}$ con 18 pazienti con pattern $10 \mathrm{kD}$. Una insufficienza renale cronica (definita come creatinina serica irreversibilmente superiore a 1.4 $\mathrm{mg} / \mathrm{dl}$ con un aumento superiore al $50 \%$ del livello basale) si sviluppava nel $50 \%$ dei pazienti con pattern $10 \mathrm{kD}$ e nel $12.5 \%$ dei pazienti con pattern $23 \mathrm{kD}$ con una differenza altamente significativa tra le curve di sopravvivenza $(\mathrm{p}=0.0001)$, anche dopo stratificazione per sindrome nefrosica $(\mathrm{p}=0.0002)$ (Tab. III). In una analisi retrospettiva di 20 pazienti con sindrome nefrosica e funzione renale normale (GSF 13 e GNM 7 pazienti) trattati con steroidi (13 pazienti) o con steroidi e ciclofosfamide (7 pazienti), l' $80 \%$ dei pazienti con pattern $23 \mathrm{kD}$ e il $30 \%$ dei pazienti con pattern $10 \mathrm{kD}$ erano responsivi alla terapia con una differenza statisticamente significativa $(\mathrm{p}=0.025)$ (la responsività era definita come remissione clinica completa o parziale o riduzione della proteinuria a livelli inferiori al $50 \%$ di quelli basali con persistenza di una funzionalità renale normale).

In ulteriori studi che hanno preso in considerazione distintamente casistiche di pazienti con GSF o GNM $(25,26)$, Bazzi et al hanno confermato l'associazione del pattern misto con componente tubulare più severa (pattern $10 \mathrm{kD}$ ) con la gravità del danno tubulo interstiziale; così come è stato confermato il maggior valore predittivo del pattern $10 \mathrm{kD}$ nei confronti del pattern $23 \mathrm{kD}$ rispetto alla progressione alla insufficienza renale cronica. In 21 pazienti con GSF, sindrome nefrosica e funzione renale basale normale ( $\mathrm{sCr} 0.91 \pm 0.18 \mathrm{mg} / \mathrm{dl}$ ) (follow up di $40 \pm 25$ mesi), il $40 \%$ dei pazienti $10 \mathrm{kD}$ e il $9 \%$ dei pazienti $23 \mathrm{kD}$ sviluppava una insufficienza renale cronica $(\mathrm{p}=0.019)$ (25) (Tab. III). In 34 pazienti

TAB. III - VALORE PREDITTIVO RISPETTO ALLA PROGRESSIONE ALLA IRC DEI PATTERN PROTEINURICI SDS-PAGE IN ALCUNE GLOMERULONEFRITI PRIMITIVE

IRC p

\begin{tabular}{|c|c|c|c|}
\hline Proteine LMW presenti vs assenti & $47 \%$ & $10 \%$ & 0.001 \\
\hline \multicolumn{4}{|l|}{ GSF, GNM, GMP (24) } \\
\hline Pattern $10 \mathrm{kD}$ vs $23 \mathrm{kD}$ & $50 \%$ & $12 \%$ & 0.0001 \\
\hline \multicolumn{4}{|l|}{ GSF (25) } \\
\hline Pattern $10 \mathrm{kD}$ vs $23 \mathrm{kD} *$ & $40 \%$ & $9 \%$ & 0.019 \\
\hline \multicolumn{4}{|l|}{ GNM (26) } \\
\hline Pattern $10 \mathrm{kD}$ vs $23 \mathrm{kD} *$ & $44 \%$ & $20 \%$ & 0.002 \\
\hline
\end{tabular}

con GNM (26), sindrome nefrosica e funzione renale basale normale $(\mathrm{sCr}$ $0.98 \pm 0.22 \mathrm{mg} / \mathrm{dl}$ ) (follow up $46 \pm 27 \mathrm{me}-$ si) una insufficienza renale cronica si sviluppava nel $44 \%$ dei pazienti con pattern $10 \mathrm{kD}$ e nel $20 \%$ dei pazienti con pattern $23 \mathrm{kD}$, con una differenza statisticamente significativa $(\mathrm{p}=0.002)$ (Tab. III). Limitatamente alla GSF Bazzi et al (25) hanno anche confermato il valore predittivo dei pattern proteinurici rispetto alla terapia: in 20 pazienti con GSF, dei quali 8 trattati con steroidi e 12 con steroidi e ciclofosfamide, una risposta alla terapia (definita come sopra indicato) si osservava nel $90 \%$ dei pazienti $23 \mathrm{kD}$ e nel $40 \%$ dei pazienti $10 \mathrm{kD}(\mathrm{p}=0.019)$. Bazzi et al (27) hanno anche valutato il valore predittivo rispetto alla progressione alla insufficienza renale cronica del pattern proteinurico SDS-PAGE associato alla presenza o meno nelle urine di polimeri della albumina urinaria, evidenziati mediante SDS-PAGE seguita da immunoblotting con antisieri anti-albumina umana in una casistica di 142 pazienti [GNLM (15), GSF (27), GNM (47) e GNMP (20) e IgAN (33)]. Polimeri della albumina urinaria erano pre- senti nell' $87 \%$ dei pazienti con GNLM, nel $52 \%$ dei pazienti con GSF, nel $51 \%$ dei pazienti con GNM, nel $55 \%$ dei pazienti con GNMP e solo nel $9 \%$ dei pazienti con IgAN (e in particolare solo in pazienti con sindrome nefrosica) con una associazione statisticamente significativa $(\mathrm{p}=0.000)$ con le forme di GN caratterizzate da lesioni prevalenti della parete del capillare glomerulare. La presenza dei polimeri era inoltre associata alla presenza di sindrome nefrosica $(\mathrm{p}=0.000)$ e alla entità della proteinuria $(\mathrm{p}=0.001)$, mentre nessuna associazione si osservava tra presenza dei polimeri e parametri istologici come la sclerosi glomerulare, il danno tubulo-interstiziale e la ialinosi arteriolare. In base a questo tipo di associazioni, i polimeri della albumina urinaria possono essere considerati un marker di danno della parete del capillare glomerulare. In 72 pazienti (22 GSF, 36 GNM, 14 GNMP) con funzionalità renale basale normale $(\mathrm{sCr} 1.02 \pm 0.23 \mathrm{mg} / \mathrm{dl})$ e un follow up di $52 \pm 27$ mesi, la presenza dei polimeri di albumina era significativamente associata alla progressione alla IRC (presenza dei polimeri: IRC 36\%; assenza dei polimeri: IRC 9\%; $\mathrm{p}=0.007$ ). 
Molto interessante era l'osservazione che la presenza combinata di un marker di danno della parete del capillare glomerulare (polimeri di albumina) e di un marker di danno tubulo-interstiziale (pattern SDS-PAGE $10 \mathrm{kD}$ ) aveva un valore predittivo rispetto alla IRC superiore a quello dei 2 marker proteinurici considerati isolatamente: presenza dei polimeri di albumina + pattern $10 \mathrm{kD}$ : IRC $62 \%$; assenza dei polimeri di albumina + pattern $23 \mathrm{kD}$ : IRC 13\%; $\mathrm{p}=0.0001$ ). Limitando lo studio ai pazienti con sindrome nefrosica, una insufficienza renale cronica si sviluppava nel $70 \%$ dei pazienti con polimeri di albumina e pattern $10 \mathrm{kD}$ e nel $14 \%$ dei pazienti con assenza dei polimeri di albumina e pattern $23 \mathrm{kD}$ $(\mathrm{p}=0.001)$. In un piccolo numero di pazienti studiati retrospettivamente rispetto alla risposta alla terapia la combinazione dei polimeri di albumina con il pattern $10 \mathrm{kD}$ aveva un valore predittivo rispetto alla non responsività al trattamento superiore a quello dei 2 markers considerati isolatamente.

\section{Conclusioni}

Nelle glomerulonefriti la caratterizzazione della proteinuria mediante elettroforesi in gel di poliacrilamide, che separa le miscele di proteine in base al loro peso molecolare, consente di distinguere $\mathrm{i}$ pazienti con proteinuria glomerulare pura da quelli con proteinuria mista glomerulo-tubulare e nell'ambito delle proteinurie miste consente di definire quadri con componente tubulare di diversa gravità. Il significato clinico dei pattern con componente tubulare più severa è abbastanza omogeneo nelle varie forme di glomerulonefrite primitiva che sono state finora studiate (IgAN, GSF, GNM) nonostante che la definizione dei pattern sia stata diversa tra i vari Autori: il pattern con componente tubulare più severa è risultato associato a livelli più elevati di creatininemia e di proteinuria totale e soprattutto ad un grado più marcato di danno tubulo-interstiziale e a una più frequente associazione con la progressione alla insufficienza renale cronica. È stato quindi confermato mediante un approccio non istologico il ruolo che il danno tubulo-interstiziale riveste rispetto alla progressione nelle principali e più frequenti forme di glomerulonefriti primitive. Limitatamente alla GSF è stato anche evidenziato un valore predittivo della componente tubulare della proteinuria rispetto alla risposta alla terapia. Pertanto l'elettroforesi in gel di poliacrilamide può essere considerata un utile sussidio diagnostico nell'inquadramento delle glomerulonefriti, in quanto può contribuire al chiarimento dei meccanismi patogenetici di progressione e consentire di individuare sottogruppi di pazienti con diversa tendenza alla progressione e diversa risposta alla terapia.

\section{BIBLIOGRAFIA}

1. Cameron JS. Proteinuria and progresson in human glomerular diseases. Am J Nephrol 1990; 10: 81-87.

2. Williams JD, Coles GA. Proteinuria - A direct cause of renal morbidity? Kidney Int 1994; 45: 443-50.

3. D'Amico G. Influence of clinical and histological features on actuarial renal survival in adult patients with idiopathic IgA Nephropathy, Membranous Nephropathy and Membranoproliferative Glomerulonephritis: survey of the recent literature. Am J Kidney Dis 1992; 20: 315-23.

4. Remuzzi G, Bertani T. Is glomerulosclerosis a consequence of altered glomeular permeability to macromolecules? Kidney Int 1990; 38: 384-94.

5. Fine LG, Ong ACM, Norman JT. Mechanisms of tubulo-interstitial injury in progressive renal disease. Eur J Clin Invest 1993; 23: 259265.

6. Ong ACM, Fine LG. Loss of glomerular function and tubulointerstitial fibrosis: cause or effect? Kidney Intern 1994; 45: 345-351.

7. Nath KA. Tubulointerstitial changes as a major determinant in the progression of renal damage. Am J Kidney Dis 1992; 20: 1-17.

8. D'Amico G, Ferrario F, Rastaldi MP. Tubulointerstitial damage in glomerular diseases: its role in the progression of renal damage. Am J Kidney Dis 1995; 26: 124-32.

9. Whermann M, Bohle A, Held H, Schumm G, Kendziorra H, Pressler H. Long-term prognosis of focal sclerosing glomerulonephritis. An analysis of 250 cases with particular regard to tubulointerstitial changes. Clin Nephrol 1990; 33: 115-122.

10. Korbet SM, Schwartz MM, Lewis EJ. The prognosis of focal segmental glomerular sclerosis 
of adulthood. Medicine 1986; 65: 304-311.

11. Myers BD, Guasch A. Mechanism of massive proteinuria. J Nephrol 1994; 7: 254-260.

12. Remuzzi A, Remuzzi G. Glomerular perm-selective function. Kidney Int 1994; 45: 398-402.

13. Carone FA, Peterson DR, Oparil S, Pullman TN. Renal tubular transport and catabolism of proteins and peptides. Kidney Int 1979; 16: 271-278.

14. Maack T, Johnson V, Kau ST, Figueiredo J, Sigulem D. Renal Filtration, transport and metabolism of low-molecular weight proteins. A review. Kidney Int 1979; 16: 25170.

15. Pesce AJ, Boreisha I, Pollack VE. Rapid differentiation of glomerular and tubular proteinuria by sodium dodecyl sulfate polyacrylamide gel electrophoresis. Clin Chim Acta 1972; 40: 27-34.

16. Lison AE, Doedt E, Meyer E. Automatic microdisc electrophoresis for urinary protein analysis: a comparison with standard analysis. Clin Nephrol 1989; 32: 40-45.

17. Stierle HE, Oser B, Boesken WH. Improved classification of proteinuria by semiautomated ultrathin SDS polyacrylamid gel electrophoresis. Clin Nephrol 1990; 33: 168173.

18. Laemmly UK. Cleavage of structural proteins during the assembly of the head of bacterophage T4. Nature 1970; 227: 680-685.

19. Petrini C, Rizza V, Bazzi C. Moderni metodi di analisi e caratterizzazione della proteinuria. In: D’Amico G, Bazzi C, Colasanti G. Attualità nefrologiche e dialitiche '94. Ed Wichtig, Milano 1995; 161169.

20. Pires MT, Da Cunha AS, Virella G, Simoes J. Analytical characterization of urinary proteins by sodium dodecyl sulphate-polyacrilamide gel electrophoresis in renal disease. Nephron 1975; 14: 361-372.
21. Nagy J, Miltenyi M, Dobos M, Burger T. Tubular proteinuria in IgA glomerulonephritis. Clin Nephrol 1987; 27: 76-78.

22. Woo KT, Lau YK, Lee GSL, Wei SS, Lim CH. Pattern of proteinuria in $\operatorname{IgA}$ nephritis by SDS-PAGE: clinical significance. Clin Nephrol 1991; 36: 6-11.

23. Bazzi C, Petrini C, Rizza V et al. SDS-PAGE patterns and polymeric albumin in proteinuria of lupus glomerulonephritis. Clin Nephrol 1995; 43: 96-103.

24. Bazzi C, Petrini C, Rizza V, Arrigo G, Beltrame A, D'Amico G. Characterization of proteinuria in primary glomerulonephritides. SDSPAGE patterns: clinical significance and prognostic value of low molecular weight ("Tubular") proteins. Am J Kid Dis 1997; 29: 27-35.

25. Bazzi C, Petrini C, Rizza V Arrigo G, Beltrame A, D'Amico G. Pattern proteinurico SDS-PAGE nella GNLM e nella GSF: differenze e valore predittivo rispetto alla IRC e alla risposta alla terapia (Abstract). Giorn It Nefrol 1997; 14 (S-9): 1.

26. Bazzi C, Petrini C, Rizza V, Arrigo G, Beltrame A, D’Amico G. Valore predittivo rispetto alla IRC e alla risposta alla terapia dei pattern proteinurici SDS-PAGE in 80 pazienti con GNM (Abstract). Giorn It Nefrol 1997; 14 (S-9): 2.

27. Bazzi C, Petrini C, Rizza V, Arrigo G, Beltrame A, D'Amico G. Characterization of proteinuria in primary glomerulonephritides: urinary polymers of albumin. Am J Kid Dis 1997; 30: 404-412. 